\title{
PVTx Measurements of the N-Methylpyrrolidone/Methanol Mixed Solvent. Cubic and SAFT EOS Analyses
}

\author{
Santiago Aparicio, Begoña García, Rafael Alcalde, María J. Dávila and José M. Leal* \\ Universidad de Burgos, Departamento de Química, 09001 Burgos, Spain
}

Corresponding Author: jmleal@ubu.es

Supporting Information 
Table I. Experimental densities, $\rho$, for the $x$ NMP + $(1-x)$ methanol binary solvents as a function of pressure and temperature

\begin{tabular}{|c|c|c|c|c|c|c|c|c|c|}
\hline \multirow[b]{2}{*}{$P(\mathrm{MPa})$} & \multicolumn{9}{|c|}{$T(\mathrm{~K})$} \\
\hline & 278.15 & 288.15 & 298.15 & 308.15 & 318.15 & 328.15 & 338.15 & 348.15 & 358.15 \\
\hline & \multicolumn{9}{|c|}{$x=0.0000$} \\
\hline 0.10 & 0.8065 & 0.7972 & 0.7876 & 0.7780 & 0.7686 & 0.7583 & 0.7487 & 0.7385 & 0.7280 \\
\hline 1.00 & 0.8073 & 0.7981 & 0.7885 & 0.7789 & 0.7696 & 0.7594 & 0.7498 & 0.7398 & 0.7293 \\
\hline 5.00 & 0.8109 & 0.8019 & 0.7925 & 0.7831 & 0.7740 & 0.7641 & 0.7548 & 0.7451 & 0.7350 \\
\hline 10.00 & 0.8152 & 0.8063 & 0.7972 & 0.7880 & 0.7792 & 0.7696 & 0.7606 & 0.7513 & 0.7416 \\
\hline 15.00 & 0.8192 & 0.8106 & 0.8021 & 0.7927 & 0.7842 & 0.7747 & 0.7661 & 0.7571 & 0.7477 \\
\hline 20.00 & 0.8231 & 0.8147 & 0.8060 & 0.7972 & 0.7888 & 0.7797 & 0.7712 & 0.7625 & 0.7535 \\
\hline 25.00 & 0.8269 & 0.8186 & 0.8100 & 0.8015 & 0.7932 & 0.7843 & 0.7761 & 0.7676 & 0.7588 \\
\hline 30.00 & 0.8305 & 0.8223 & 0.8139 & 0.8055 & 0.7975 & 0.7888 & 0.7807 & 0.7724 & 0.7638 \\
\hline 35.00 & 0.8340 & 0.8260 & 0.8177 & 0.8094 & 0.8016 & 0.7930 & 0.7851 & 0.7770 & 0.7686 \\
\hline 40.00 & 0.8373 & 0.8295 & 0.8213 & 0.8131 & 0.8054 & 0.7971 & 0.7893 & 0.7814 & 0.7732 \\
\hline 45.00 & 0.8406 & 0.8328 & 0.8249 & 0.8168 & 0.8091 & 0.8010 & 0.7934 & 0.7856 & 0.7776 \\
\hline 50.00 & 0.8438 & 0.8361 & 0.8282 & 0.8203 & 0.8128 & 0.8047 & 0.7973 & 0.7897 & 0.7818 \\
\hline 55.00 & 0.8468 & 0.8393 & 0.8315 & 0.8236 & 0.8163 & 0.8084 & 0.8011 & 0.7936 & 0.7859 \\
\hline \multirow[t]{2}{*}{60.00} & 0.8497 & 0.8421 & 0.8345 & 0.8267 & 0.8197 & 0.8119 & 0.8047 & 0.7973 & 0.7896 \\
\hline & \multicolumn{9}{|c|}{$x=0.0972$} \\
\hline 0.10 & 0.8611 & 0.8519 & 0.8423 & 0.8328 & 0.8234 & 0.8135 & 0.8039 & 0.7938 & 0.7835 \\
\hline 1.00 & 0.8617 & 0.8526 & 0.8431 & 0.8335 & 0.8242 & 0.8144 & 0.8048 & 0.7949 & 0.7846 \\
\hline 5.00 & 0.8648 & 0.8558 & 0.8465 & 0.8371 & 0.8280 & 0.8184 & 0.8092 & 0.7995 & 0.7895 \\
\hline 10.00 & 0.8685 & 0.8597 & 0.8506 & 0.8415 & 0.8326 & 0.8232 & 0.8142 & 0.8048 & 0.7952 \\
\hline 15.00 & 0.8721 & 0.8635 & 0.8549 & 0.8456 & 0.8369 & 0.8277 & 0.8190 & 0.8099 & 0.8005 \\
\hline 20.00 & 0.8755 & 0.8670 & 0.8583 & 0.8495 & 0.8410 & 0.8321 & 0.8235 & 0.8146 & 0.8056 \\
\hline 25.00 & 0.8789 & 0.8705 & 0.8619 & 0.8533 & 0.8450 & 0.8362 & 0.8278 & 0.8192 & 0.8104 \\
\hline 30.00 & 0.8822 & 0.8739 & 0.8655 & 0.8569 & 0.8488 & 0.8401 & 0.8319 & 0.8235 & 0.8149 \\
\hline 35.00 & 0.8852 & 0.8771 & 0.8688 & 0.8604 & 0.8525 & 0.8439 & 0.8359 & 0.8277 & 0.8193 \\
\hline 40.00 & 0.8883 & 0.8803 & 0.8721 & 0.8638 & 0.8560 & 0.8476 & 0.8397 & 0.8317 & 0.8235 \\
\hline 45.00 & 0.8912 & 0.8834 & 0.8753 & 0.8671 & 0.8594 & 0.8512 & 0.8434 & 0.8356 & 0.8275 \\
\hline 50.00 & 0.8941 & 0.8863 & 0.8783 & 0.8703 & 0.8627 & 0.8546 & 0.8471 & 0.8393 & 0.8314 \\
\hline 55.00 & 0.8969 & 0.8892 & 0.8814 & 0.8734 & 0.8659 & 0.8580 & 0.8506 & 0.8430 & 0.8352 \\
\hline \multirow[t]{2}{*}{60.00} & 0.8994 & 0.8918 & 0.8841 & 0.8762 & 0.8690 & 0.8612 & 0.8539 & 0.8464 & 0.8386 \\
\hline & \multicolumn{9}{|c|}{$x=0.1963$} \\
\hline 0.10 & 0.9030 & 0.8938 & 0.8844 & 0.8749 & 0.8655 & 0.8558 & 0.8462 & 0.8363 & 0.8261 \\
\hline 1.00 & 0.9037 & 0.8945 & 0.8851 & 0.8756 & 0.8663 & 0.8566 & 0.8471 & 0.8373 & 0.8271 \\
\hline 5.00 & 0.9064 & 0.8974 & 0.8882 & 0.8788 & 0.8697 & 0.8602 & 0.8509 & 0.8413 & 0.8314 \\
\hline 10.00 & 0.9098 & 0.9009 & 0.8918 & 0.8827 & 0.8738 & 0.8645 & 0.8554 & 0.8460 & 0.8365 \\
\hline 15.00 & 0.9131 & 0.9044 & 0.8957 & 0.8865 & 0.8778 & 0.8686 & 0.8598 & 0.8507 & 0.8414 \\
\hline 20.00 & 0.9161 & 0.9077 & 0.8989 & 0.8901 & 0.8816 & 0.8725 & 0.8639 & 0.8551 & 0.8461 \\
\hline 25.00 & 0.9192 & 0.9108 & 0.9022 & 0.8935 & 0.8851 & 0.8763 & 0.8679 & 0.8592 & 0.8505 \\
\hline 30.00 & 0.9221 & 0.9139 & 0.9054 & 0.8968 & 0.8886 & 0.8799 & 0.8717 & 0.8632 & 0.8546 \\
\hline 35.00 & 0.9250 & 0.9168 & 0.9085 & 0.9000 & 0.8920 & 0.8834 & 0.8754 & 0.8671 & 0.8586 \\
\hline 40.00 & 0.9278 & 0.9198 & 0.9115 & 0.9031 & 0.8952 & 0.8869 & 0.8789 & 0.8707 & 0.8625 \\
\hline 45.00 & 0.9305 & 0.9226 & 0.9145 & 0.9062 & 0.8984 & 0.8902 & 0.8823 & 0.8744 & 0.8662 \\
\hline 50.00 & 0.9332 & 0.9253 & 0.9173 & 0.9092 & 0.9015 & 0.8934 & 0.8857 & 0.8779 & 0.8699 \\
\hline 55.00 & 0.9357 & 0.9280 & 0.9201 & 0.9121 & 0.9045 & 0.8965 & 0.8890 & 0.8812 & 0.8734 \\
\hline 60.00 & 0.9381 & 0.9304 & 0.9226 & 0.9147 & 0.9073 & 0.8995 & 0.8920 & 0.8844 & 0.8766 \\
\hline
\end{tabular}


Table I. Continued

\begin{tabular}{|c|c|c|c|c|c|c|c|c|c|}
\hline \multirow[b]{2}{*}{$P(\mathrm{MPa})$} & \multicolumn{9}{|c|}{$T(\mathrm{~K})$} \\
\hline & 278.15 & 288.15 & 298.15 & 308.15 & 318.15 & 328.15 & 338.15 & 348.15 & 358.15 \\
\hline & \multicolumn{9}{|c|}{$x=0.2933$} \\
\hline 0.10 & 0.9349 & 0.9257 & 0.9164 & 0.9069 & 0.8975 & 0.8879 & 0.8784 & 0.8685 & 0.8587 \\
\hline 1.00 & 0.9354 & 0.9263 & 0.9171 & 0.9075 & 0.8982 & 0.8886 & 0.8792 & 0.8694 & 0.8596 \\
\hline 5.00 & 0.9380 & 0.9290 & 0.9198 & 0.9105 & 0.9014 & 0.8920 & 0.8828 & 0.8732 & 0.8635 \\
\hline 10.00 & 0.9411 & 0.9322 & 0.9232 & 0.9141 & 0.9052 & 0.8959 & 0.8869 & 0.8776 & 0.8682 \\
\hline 15.00 & 0.9441 & 0.9354 & 0.9268 & 0.9176 & 0.9089 & 0.8997 & 0.8910 & 0.8819 & 0.8727 \\
\hline 20.00 & 0.9470 & 0.9384 & 0.9297 & 0.9209 & 0.9124 & 0.9034 & 0.8948 & 0.8860 & 0.8770 \\
\hline 25.00 & 0.9498 & 0.9414 & 0.9328 & 0.9242 & 0.9158 & 0.9070 & 0.8985 & 0.8899 & 0.8811 \\
\hline 30.00 & 0.9525 & 0.9443 & 0.9358 & 0.9273 & 0.9191 & 0.9104 & 0.9021 & 0.8936 & 0.8850 \\
\hline 35.00 & 0.9553 & 0.9471 & 0.9387 & 0.9303 & 0.9222 & 0.9136 & 0.9056 & 0.8972 & 0.8888 \\
\hline 40.00 & 0.9579 & 0.9498 & 0.9416 & 0.9332 & 0.9253 & 0.9169 & 0.9089 & 0.9007 & 0.8925 \\
\hline 45.00 & 0.9604 & 0.9525 & 0.9444 & 0.9361 & 0.9282 & 0.9200 & 0.9122 & 0.9041 & 0.8961 \\
\hline 50.00 & 0.9630 & 0.9551 & 0.9471 & 0.9390 & 0.9312 & 0.9231 & 0.9154 & 0.9075 & 0.8996 \\
\hline 55.00 & 0.9654 & 0.9577 & 0.9497 & 0.9417 & 0.9341 & 0.9261 & 0.9185 & 0.9107 & 0.9029 \\
\hline \multirow[t]{2}{*}{60.00} & 0.9677 & 0.9600 & 0.9522 & 0.9443 & 0.9368 & 0.9290 & 0.9214 & 0.9138 & 0.9060 \\
\hline & \multicolumn{9}{|c|}{$x=0.3939$} \\
\hline 0.10 & 0.9610 & 0.9519 & 0.9427 & 0.9333 & 0.9241 & 0.9145 & 0.9052 & 0.8955 & 0.8856 \\
\hline 1.00 & 0.9616 & 0.9525 & 0.9433 & 0.9339 & 0.9248 & 0.9153 & 0.9059 & 0.8963 & 0.8864 \\
\hline 5.00 & 0.9639 & 0.9551 & 0.9459 & 0.9368 & 0.9277 & 0.9184 & 0.9093 & 0.8998 & 0.8901 \\
\hline 10.00 & 0.9669 & 0.9581 & 0.9491 & 0.9401 & 0.9313 & 0.9221 & 0.9132 & 0.9040 & 0.8946 \\
\hline 15.00 & 0.9697 & 0.9611 & 0.9525 & 0.9434 & 0.9348 & 0.9256 & 0.9169 & 0.9079 & 0.8988 \\
\hline 20.00 & 0.9725 & 0.9640 & 0.9553 & 0.9465 & 0.9380 & 0.9291 & 0.9206 & 0.9118 & 0.9029 \\
\hline 25.00 & 0.9752 & 0.9668 & 0.9583 & 0.9496 & 0.9412 & 0.9325 & 0.9241 & 0.9154 & 0.9068 \\
\hline 30.00 & 0.9778 & 0.9695 & 0.9611 & 0.9525 & 0.9443 & 0.9357 & 0.9275 & 0.9190 & 0.9105 \\
\hline 35.00 & 0.9803 & 0.9722 & 0.9638 & 0.9554 & 0.9473 & 0.9388 & 0.9308 & 0.9224 & 0.9141 \\
\hline 40.00 & 0.9828 & 0.9747 & 0.9665 & 0.9581 & 0.9502 & 0.9419 & 0.9339 & 0.9257 & 0.9175 \\
\hline 45.00 & 0.9852 & 0.9773 & 0.9692 & 0.9609 & 0.9530 & 0.9448 & 0.9370 & 0.9290 & 0.9209 \\
\hline 50.00 & 0.9876 & 0.9797 & 0.9717 & 0.9636 & 0.9558 & 0.9477 & 0.9400 & 0.9321 & 0.9242 \\
\hline 55.00 & 0.9899 & 0.9821 & 0.9742 & 0.9662 & 0.9585 & 0.9505 & 0.9430 & 0.9352 & 0.9274 \\
\hline \multirow[t]{2}{*}{60.00} & 0.9920 & 0.9843 & 0.9765 & 0.9686 & 0.9611 & 0.9532 & 0.9457 & 0.9381 & 0.9304 \\
\hline & \multicolumn{9}{|c|}{$x=0.4910$} \\
\hline 0.10 & 0.9813 & 0.9722 & 0.9630 & 0.9538 & 0.9447 & 0.9354 & 0.9261 & 0.9164 & 0.9068 \\
\hline 1.00 & 0.9818 & 0.9728 & 0.9636 & 0.9544 & 0.9454 & 0.9360 & 0.9267 & 0.9171 & 0.9076 \\
\hline 5.00 & 0.9841 & 0.9752 & 0.9661 & 0.9571 & 0.9482 & 0.9390 & 0.9299 & 0.9205 & 0.9111 \\
\hline 10.00 & 0.9869 & 0.9781 & 0.9692 & 0.9603 & 0.9515 & 0.9425 & 0.9336 & 0.9244 & 0.9152 \\
\hline 15.00 & 0.9896 & 0.9810 & 0.9724 & 0.9634 & 0.9548 & 0.9459 & 0.9372 & 0.9282 & 0.9192 \\
\hline 20.00 & 0.9922 & 0.9837 & 0.9751 & 0.9664 & 0.9580 & 0.9492 & 0.9406 & 0.9318 & 0.9231 \\
\hline 25.00 & 0.9948 & 0.9864 & 0.9779 & 0.9693 & 0.9610 & 0.9524 & 0.9441 & 0.9354 & 0.9269 \\
\hline 30.00 & 0.9972 & 0.9891 & 0.9807 & 0.9722 & 0.9640 & 0.9555 & 0.9473 & 0.9389 & 0.9305 \\
\hline 35.00 & 0.9997 & 0.9916 & 0.9833 & 0.9750 & 0.9669 & 0.9586 & 0.9505 & 0.9422 & 0.9339 \\
\hline 40.00 & 1.0021 & 0.9941 & 0.9859 & 0.9776 & 0.9697 & 0.9615 & 0.9535 & 0.9454 & 0.9373 \\
\hline 45.00 & 1.0044 & 0.9965 & 0.9885 & 0.9803 & 0.9724 & 0.9643 & 0.9565 & 0.9485 & 0.9405 \\
\hline 50.00 & 1.0067 & 0.9989 & 0.9909 & 0.9828 & 0.9751 & 0.9671 & 0.9594 & 0.9515 & 0.9437 \\
\hline 55.00 & 1.0089 & 1.0012 & 0.9933 & 0.9853 & 0.9777 & 0.9698 & 0.9622 & 0.9545 & 0.9467 \\
\hline 60.00 & 1.0110 & 1.0033 & 0.9956 & 0.9877 & 0.9802 & 0.9724 & 0.9649 & 0.9572 & 0.9496 \\
\hline
\end{tabular}


Table I. Continued

\begin{tabular}{|c|c|c|c|c|c|c|c|c|c|}
\hline \multirow[b]{2}{*}{$P(\mathrm{MPa})$} & \multicolumn{9}{|c|}{$T(\mathrm{~K})$} \\
\hline & 278.15 & 288.15 & 298.15 & 308.15 & 318.15 & 328.15 & 338.15 & 348.15 & 358.15 \\
\hline & \multicolumn{9}{|c|}{$x=0.5986$} \\
\hline 0.10 & 0.9998 & 0.9909 & 0.9818 & 0.9726 & 0.9637 & 0.9544 & 0.9453 & 0.9358 & 0.9263 \\
\hline 1.00 & 1.0003 & 0.9914 & 0.9824 & 0.9732 & 0.9643 & 0.9551 & 0.9459 & 0.9365 & 0.9271 \\
\hline 5.00 & 1.0026 & 0.9939 & 0.9848 & 0.9758 & 0.9670 & 0.9579 & 0.9489 & 0.9396 & 0.9304 \\
\hline 10.00 & 1.0053 & 0.9967 & 0.9879 & 0.9788 & 0.9702 & 0.9612 & 0.9524 & 0.9434 & 0.9343 \\
\hline 15.00 & 1.0079 & 0.9994 & 0.9908 & 0.9819 & 0.9734 & 0.9645 & 0.9559 & 0.9470 & 0.9382 \\
\hline 20.00 & 1.0104 & 1.0021 & 0.9935 & 0.9848 & 0.9764 & 0.9677 & 0.9592 & 0.9505 & 0.9419 \\
\hline 25.00 & 1.0129 & 1.0046 & 0.9962 & 0.9876 & 0.9793 & 0.9707 & 0.9624 & 0.9539 & 0.9454 \\
\hline 30.00 & 1.0153 & 1.0071 & 0.9988 & 0.9904 & 0.9822 & 0.9737 & 0.9656 & 0.9572 & 0.9488 \\
\hline 35.00 & 1.0176 & 1.0096 & 1.0013 & 0.9930 & 0.9850 & 0.9766 & 0.9686 & 0.9603 & 0.9521 \\
\hline 40.00 & 1.0199 & 1.0120 & 1.0038 & 0.9956 & 0.9877 & 0.9795 & 0.9715 & 0.9634 & 0.9553 \\
\hline 45.00 & 1.0222 & 1.0143 & 1.0063 & 0.9981 & 0.9903 & 0.9822 & 0.9744 & 0.9664 & 0.9585 \\
\hline 50.00 & 1.0244 & 1.0166 & 1.0087 & 1.0007 & 0.9929 & 0.9849 & 0.9773 & 0.9694 & 0.9616 \\
\hline 55.00 & 1.0266 & 1.0189 & 1.0110 & 1.0031 & 0.9955 & 0.9876 & 0.9800 & 0.9723 & 0.9646 \\
\hline \multirow[t]{2}{*}{60.00} & 1.0286 & 1.0210 & 1.0132 & 1.0054 & 0.9978 & 0.9901 & 0.9826 & 0.9749 & 0.9674 \\
\hline & \multicolumn{9}{|c|}{$x=0.6985$} \\
\hline 0.10 & 1.0139 & 1.0050 & 0.9961 & 0.9869 & 0.9781 & 0.9689 & 0.9599 & 0.9505 & 0.9412 \\
\hline 1.00 & 1.0143 & 1.0055 & 0.9966 & 0.9874 & 0.9786 & 0.9695 & 0.9604 & 0.9512 & 0.9419 \\
\hline 5.00 & 1.0164 & 1.0078 & 0.9989 & 0.9899 & 0.9812 & 0.9721 & 0.9633 & 0.9542 & 0.9451 \\
\hline 10.00 & 1.0191 & 1.0105 & 1.0017 & 0.9928 & 0.9843 & 0.9754 & 0.9667 & 0.9578 & 0.9488 \\
\hline 15.00 & 1.0216 & 1.0132 & 1.0046 & 0.9958 & 0.9873 & 0.9785 & 0.9700 & 0.9612 & 0.9525 \\
\hline 20.00 & 1.0241 & 1.0157 & 1.0073 & 0.9986 & 0.9903 & 0.9817 & 0.9733 & 0.9646 & 0.9561 \\
\hline 25.00 & 1.0265 & 1.0182 & 1.0099 & 1.0013 & 0.9931 & 0.9846 & 0.9764 & 0.9679 & 0.9595 \\
\hline 30.00 & 1.0288 & 1.0207 & 1.0124 & 1.0040 & 0.9959 & 0.9876 & 0.9794 & 0.9711 & 0.9628 \\
\hline 35.00 & 1.0311 & 1.0231 & 1.0149 & 1.0066 & 0.9986 & 0.9904 & 0.9824 & 0.9742 & 0.9661 \\
\hline 40.00 & 1.0334 & 1.0254 & 1.0173 & 1.0091 & 1.0013 & 0.9931 & 0.9853 & 0.9771 & 0.9692 \\
\hline 45.00 & 1.0356 & 1.0277 & 1.0197 & 1.0116 & 1.0038 & 0.9958 & 0.9881 & 0.9801 & 0.9722 \\
\hline 50.00 & 1.0377 & 1.0300 & 1.0221 & 1.0140 & 1.0064 & 0.9984 & 0.9908 & 0.9830 & 0.9753 \\
\hline 55.00 & 1.0398 & 1.0322 & 1.0243 & 1.0164 & 1.0088 & 1.0010 & 0.9935 & 0.9857 & 0.9781 \\
\hline \multirow[t]{2}{*}{60.00} & 1.0417 & 1.0342 & 1.0264 & 1.0187 & 1.0111 & 1.0034 & 0.9959 & 0.9883 & 0.9808 \\
\hline & \multicolumn{9}{|c|}{$x=0.8049$} \\
\hline 0.10 & 1.0267 & 1.0179 & 1.0091 & 1.0000 & 0.9912 & 0.9821 & 0.9731 & 0.9638 & 0.9547 \\
\hline 1.00 & 1.0271 & 1.0184 & 1.0096 & 1.0005 & 0.9918 & 0.9827 & 0.9737 & 0.9645 & 0.9553 \\
\hline 5.00 & 1.0292 & 1.0206 & 1.0118 & 1.0029 & 0.9942 & 0.9853 & 0.9765 & 0.9673 & 0.9584 \\
\hline 10.00 & 1.0317 & 1.0232 & 1.0146 & 1.0057 & 0.9973 & 0.9884 & 0.9798 & 0.9709 & 0.9620 \\
\hline 15.00 & 1.0341 & 1.0258 & 1.0173 & 1.0086 & 1.0002 & 0.9915 & 0.9830 & 0.9743 & 0.9656 \\
\hline 20.00 & 1.0365 & 1.0283 & 1.0199 & 1.0113 & 1.0030 & 0.9945 & 0.9861 & 0.9775 & 0.9691 \\
\hline 25.00 & 1.0389 & 1.0307 & 1.0224 & 1.0139 & 1.0058 & 0.9974 & 0.9892 & 0.9807 & 0.9724 \\
\hline 30.00 & 1.0412 & 1.0331 & 1.0249 & 1.0166 & 1.0085 & 1.0002 & 0.9921 & 0.9838 & 0.9757 \\
\hline 35.00 & 1.0434 & 1.0354 & 1.0273 & 1.0191 & 1.0111 & 1.0030 & 0.9950 & 0.9868 & 0.9788 \\
\hline 40.00 & 1.0456 & 1.0377 & 1.0297 & 1.0215 & 1.0137 & 1.0056 & 0.9978 & 0.9898 & 0.9819 \\
\hline 45.00 & 1.0478 & 1.0400 & 1.0320 & 1.0240 & 1.0162 & 1.0083 & 1.0006 & 0.9926 & 0.9848 \\
\hline 50.00 & 1.0499 & 1.0421 & 1.0343 & 1.0263 & 1.0187 & 1.0108 & 1.0032 & 0.9954 & 0.9878 \\
\hline 55.00 & 1.0519 & 1.0443 & 1.0365 & 1.0287 & 1.0211 & 1.0133 & 1.0058 & 0.9981 & 0.9905 \\
\hline 60.00 & 1.0538 & 1.0463 & 1.0386 & 1.0309 & 1.0233 & 1.0157 & 1.0082 & 1.0006 & 0.9932 \\
\hline
\end{tabular}


Table I. Continued

\begin{tabular}{|c|c|c|c|c|c|c|c|c|c|}
\hline \multirow[b]{2}{*}{$P(\mathrm{MPa})$} & \multicolumn{9}{|c|}{$T(\mathrm{~K})$} \\
\hline & 278.15 & 288.15 & 298.15 & 308.15 & 318.15 & 328.15 & 338.15 & 348.15 & 358.15 \\
\hline & \multicolumn{9}{|c|}{$x=0.9024$} \\
\hline 0.10 & 1.0366 & 1.0280 & 1.0192 & 1.0102 & 1.0014 & 0.9924 & 0.9835 & 0.9743 & 0.9653 \\
\hline 1.00 & 1.0371 & 1.0285 & 1.0197 & 1.0106 & 1.0020 & 0.9929 & 0.9841 & 0.9750 & 0.9659 \\
\hline 5.00 & 1.0391 & 1.0306 & 1.0219 & 1.0130 & 1.0044 & 0.9955 & 0.9868 & 0.9778 & 0.9689 \\
\hline 10.00 & 1.0415 & 1.0331 & 1.0246 & 1.0158 & 1.0073 & 0.9986 & 0.9900 & 0.9812 & 0.9725 \\
\hline 15.00 & 1.0440 & 1.0357 & 1.0272 & 1.0186 & 1.0102 & 1.0016 & 0.9932 & 0.9845 & 0.9760 \\
\hline 20.00 & 1.0463 & 1.0381 & 1.0298 & 1.0212 & 1.0130 & 1.0046 & 0.9963 & 0.9877 & 0.9793 \\
\hline 25.00 & 1.0486 & 1.0405 & 1.0322 & 1.0238 & 1.0157 & 1.0074 & 0.9992 & 0.9908 & 0.9826 \\
\hline 30.00 & 1.0509 & 1.0428 & 1.0347 & 1.0264 & 1.0184 & 1.0102 & 1.0021 & 0.9938 & 0.9857 \\
\hline 35.00 & 1.0531 & 1.0451 & 1.0371 & 1.0289 & 1.0210 & 1.0129 & 1.0049 & 0.9968 & 0.9888 \\
\hline 40.00 & 1.0552 & 1.0474 & 1.0394 & 1.0313 & 1.0235 & 1.0155 & 1.0077 & 0.9996 & 0.9918 \\
\hline 45.00 & 1.0573 & 1.0496 & 1.0417 & 1.0337 & 1.0259 & 1.0181 & 1.0104 & 1.0024 & 0.9947 \\
\hline 50.00 & 1.0594 & 1.0517 & 1.0439 & 1.0360 & 1.0284 & 1.0205 & 1.0130 & 1.0052 & 0.9975 \\
\hline 55.00 & 1.0614 & 1.0538 & 1.0461 & 1.0383 & 1.0307 & 1.0230 & 1.0155 & 1.0078 & 1.0003 \\
\hline \multirow[t]{2}{*}{60.00} & 1.0633 & 1.0559 & 1.0481 & 1.0404 & 1.0329 & 1.0253 & 1.0178 & 1.0102 & 1.0029 \\
\hline & \multicolumn{9}{|c|}{$x=1.0000^{\mathrm{a}}$} \\
\hline 0.10 & 1.0456 & 1.0370 & 1.0282 & 1.0193 & 1.0106 & 1.0016 & 0.9928 & 0.9837 & 0.9748 \\
\hline 1.00 & 1.0461 & 1.0375 & 1.0287 & 1.0198 & 1.0111 & 1.0022 & 0.9934 & 0.9843 & 0.9754 \\
\hline 5.00 & 1.0480 & 1.0396 & 1.0309 & 1.0220 & 1.0135 & 1.0047 & 0.9960 & 0.9871 & 0.9783 \\
\hline 10.00 & 1.0504 & 1.0420 & 1.0335 & 1.0247 & 1.0164 & 1.0077 & 0.9992 & 0.9904 & 0.9818 \\
\hline 15.00 & 1.0528 & 1.0445 & 1.0361 & 1.0275 & 1.0192 & 1.0106 & 1.0023 & 0.9936 & 0.9852 \\
\hline 20.00 & 1.0551 & 1.0469 & 1.0386 & 1.0301 & 1.0219 & 1.0135 & 1.0053 & 0.9967 & 0.9884 \\
\hline 25.00 & 1.0573 & 1.0492 & 1.0410 & 1.0326 & 1.0245 & 1.0163 & 1.0081 & 0.9998 & 0.9916 \\
\hline 30.00 & 1.0595 & 1.0515 & 1.0434 & 1.0351 & 1.0271 & 1.0190 & 1.0110 & 1.0027 & 0.9947 \\
\hline 35.00 & 1.0617 & 1.0538 & 1.0457 & 1.0376 & 1.0297 & 1.0216 & 1.0137 & 1.0056 & 0.9977 \\
\hline 40.00 & 1.0638 & 1.0560 & 1.0480 & 1.0400 & 1.0322 & 1.0242 & 1.0164 & 1.0084 & 1.0006 \\
\hline 45.00 & 1.0659 & 1.0582 & 1.0503 & 1.0423 & 1.0346 & 1.0267 & 1.0190 & 1.0111 & 1.0034 \\
\hline 50.00 & 1.0680 & 1.0603 & 1.0525 & 1.0446 & 1.0370 & 1.0292 & 1.0216 & 1.0138 & 1.0062 \\
\hline 55.00 & 1.0700 & 1.0624 & 1.0546 & 1.0468 & 1.0393 & 1.0316 & 1.0241 & 1.0164 & 1.0089 \\
\hline 60.00 & 1.0718 & 1.0644 & 1.0567 & 1.0490 & 1.0414 & 1.0338 & 1.0264 & 1.0188 & 1.0115 \\
\hline
\end{tabular}

${ }^{\mathrm{a}}$ Data from Ref. 19. 